nervous system is only one out of many parts of the human frame in which this lerrible disease plays havoc, and it may be that the greater frequency and extent of its processes in this region is merely apparent-a consequence of the readiness with which the nerve-elements are deranged in their structure and function, and of the distinctness with which such derangement is revealed. Strange indeed it is to think that all this train of morbid processes, so long, 8 ) varied, often so disastrous, is the consequence, we cannot doubt, of the entrance into the system of a few organisms, scarcely to be seen even with the utmost increase in the power of vision, human ingenuity has yet contrived. And it is strange and sad to remember that this malady, rivalled in its total capacity for wrecking happiness and health and life by no other, and exceeded in apparent horror only by those whose effects are more sudden, is equally formidable by reason of our limited power over it. Make what deductions you can for the mild or latent forms of the disease, recognise all our power of repression, and the fact remains that we have yet to find the means of arresting it; and I may add we have yet to find effective means for its prevention. The philanthropy whose vision is so narrowed by misplaced feeling, that only one imperfect aspect of the means employed can be perceived, has decreed that the chief method that has been suggested shall not have so much as a trial under fair conditions, despite the manifold and varied benefit that has been obtained from its partial use-and has decreed that the disease shall continue unrestrained, to work its ruin alike on the innocent and the guilty. Against the influence of this mistaken zeal for a nominal purity and a real disease we are powerless. But there remains the fact, and it is certainly as old as the malady itself, that the disease can be prevented. One method, and one alone, is possible, and that one is open to all. It is the prevention and the safety that can be secured by unbroken chastity. Is this potentiality becoming greater? As we look back through the long centuries, we see the sensual more and more dominant in the past, growing less as the race slowly rises. But as we look at the present we can trace small ground for hope that this process will have any appreciable influence unless or until there is some change in men more potent and effective than the slow "live upward, working out the beast" of moral evolution. But that which will not perhaps be for the mass, may yet be for the individual. And in ending I must ask a question and give a warning that I would fain have left unasked, unsaid. But 1 cannot, I dare not, pass them by. Do we do all we can-and our profession gives us power that no other has-do we do all we can to promote that perfect chastity which alone can save from this, and from that which is worse? The opinions that on pseudo-physiological grounds suggest or permit incontinence are absolutely false. Trace them to their ultimate basis, and they are groundless. They rest only on sensory illusions-one of the many illustrations of a maxim I have often to enforce on various sufferers: "There are no liars like our own sensations." Or rather, I should say, they rest on misinterpretations, always biassed, and often deliberate. With all the force that any knowledge I possess can give, and with any authority I may have-as the result of long observation and consideration of the subject in all its relations-I assert that no man ever yet was in the slightest degree or way the worse for perfect continence or the better for incontinence ; that for unchastity every man must be worse morally; and that most are worse physically, and that in no small number the result is, and ever will be, utter physical shipwreck on one of the many rocks, sharp, jaggededged,' or one of the mary banks of festering slime that are about his course, and which no care can possibly avoid. My warning is : Let us beware lest we give even a silent sanction to that against which I am sure, on even the lowest grounds that we can take, we should resolutely set our face and raise our voice.

\section{ELECTRICAL STIMULATION OF THE HEART IN MAN.}

BY JOHN A. MCWILLIAM, M.D.

Professor of the Institutes of Medicine in the University of Aberdeen (From the Physiological Laboratory of the University of Aberdeen.)

IT is, of course, only in a very limited number of the cases of cardiac failure that the question of artificial excitation of the heart beat becomes one of practical importance. In the majority of instances where a more or less sudden heart stoppage occurs there are underlying conditions which obviously render direct stimulation of the organ inapplicable or hopeless. But, on the other hand, in certain forms of cardiac arrest there appears to be a possibility of restoring by artificial means the rhythmic beat, and tiding over a sudden and temporary danger. Such is especially the case in those instances where cardiac failure assumes the form of an inhibition of the heart beat by impulses reaching the organ along the vagus nerves. There is much reason to believe that among cases of sudden and fatal syncope such a mode of heart failure is not very rare. It probably obtains in many of those instances where sudden death has been caused by blows upon the præcordia or stomach (apart from the occurrence of any important visceral lesion); alse in many cases of death from fright, and, as Lauder Brunton has urged, from irritation of sensory nerves (for example, the fifth) in certain phases of the administration of chloroform. It is in regard to sudden cardiac failure from the last-mentioned cause that the question of direct excitation of the heart becomes most important. There is much probability in the view that sudden stoppage of the heart under chloroform may often be due to a sudden excitation of the cardioinhibitory centre in the medulla oblongata and a consequent arrest of the cardiac beat by inhibitory impulses carried down from the medulla by the vagus nerves; such appears to be especially probable when the heart suddenly stops, early in an operation, when a comparatively small amount of chloroform has been given and the anæsthesia may be more or less incomplete.

Now we know that when the mammalian heart has been inhibited through the vagus nerve it is quite possible to excite an immediate renewal of the rhythmic action by direct stimulation of the organ. No doubt it is very possible, as I have already suggested in a former paper, that the fate of the heart may be sealed in cases of fatal inhibitory arrest by the supervention of fibrillar contraction or heart-delirium in the ventricles. But, apart from the occurrence of such a disastrous complication, or before its inception, artificial excitation might be useful in rousing into action a heart that has been arrested by a temporary cause; for example, by inhibitory impulses profoundly depressing the rate and force of $i$ ts action, or causing it to stand still in diastole.

As regards the means to be employed for the purpose of directly stimulating the cardiac action in eases of sudden failure, various expedients have been recommended-among others the application of galvanic and faradic currents to the region of the heart, electro-puncture, mechanical irritation by a fine needle passed into the organ, and the application of heat to the præcordia.

The application of strong galvanic and faradic currents to the ventricles is attended with disastrous results ; an immediate abolition of the normal beat, and the occurrence of a wildly inco-ordinated, arhythmic contraction of the ventricular muscle (fibrillar contraction or heart-delirium), attended by a great and rapid fall of blood-pressure, and, in the higher mammals (the dog at least) by speedy death. The ventricles are utterly unable to pump out the contained blood, and the circulation quickly comes to an end.

If such a phenomenon had been observed simply in one or two sorts of animals, it might be urged that the human heart is possibly exempt from such a danger. But the phenomenon in question is one that occurs in more or less extreme form in every mammal examined, and, indeed, in birds as well. I have myself observed it in the hearts of the dog, cat, rabbit, hedgehog, rat, mouse, guinea-pig, hen, pigeon, and blackbird. It is, in all probability, of universal occurrence in the hearts of warm-blooded animals ; and it is significant that it is more easily excited and more extreme in its character in the higher mammals than in the lower.

It has been argued that the strength of current employed clinically would not be sufficient to bring about the grave result in question. In regard to this consideration there is a point of much importance, namely, that the susceptibility of the ventricular tissue to the occurrence of fibrillar contraction may become enormously augmented in certain conditions of impaired and disordered nutrition. Of this fact I have been most fully convinced in the course of my investigations on the mammalian heart, for on numerous occasions I have seen the ventricles going off into fibrillar contraction under the influence of extremely slight irritant causes, slight pressure or friction, faradic currents too weak to be felt on the tongue, etc.-causes which would have been entirely insufficient to bring about such a result in a normal, or approximately normal, heart. Now it is precisely in conditions 
of heart failure, where the nutrition of the cardiac tissues must necessarily be impaired and altered, that it is proposed to apply galvanic and faradic currents clinically. In the light of physiological experience such a procedure would seem to be liable to the gravest issues, and to be wholly unjustifiable. Of needle puncture the same must be said.

On the other hand, it is to be borne in mind that von Ziemssen, Dixon Mann, Hartmann and others have described the results they obtained from the application of electrical currents (galvanic and faradic) to the human heart, and no untoward event occurred. But these experiments were conducted on healthy persons; and, as I have already stated, the susceptibility of the normal heart is not to be taken as in any measure a test of the strength of stimulating current which can be borne without disastrous result by hearts that have suffered from a derangement of their nutritive processes, and have passed into a state of unstable equilibrium. It seems to me that the experiments of the observers above mentioned do not in any way serve as proof of the safety of galvanic and faradic currents applied to the heart; they do not deal with the organ in such conditions as those in which the clinical use of such currents is frequently recommended-conditions that physiological experience shows to be frequently associated with an immensely increased susceptibility to the occurrence of fibrillar contraction and fatal collapse of the cardiac function.

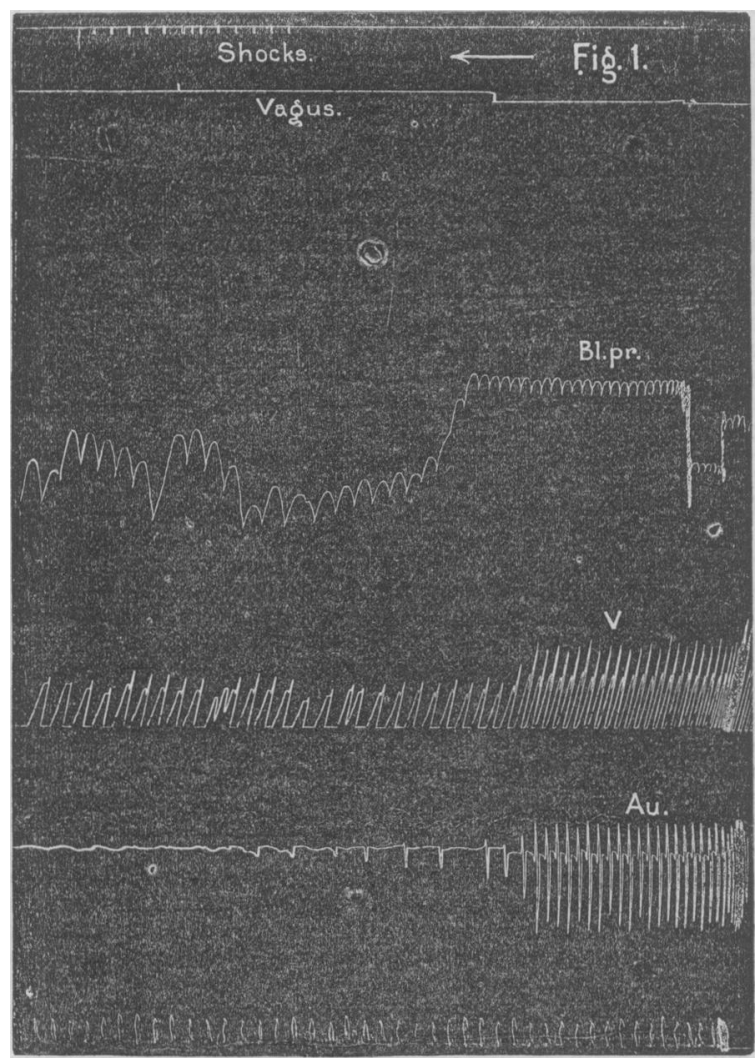

Fig. 1 (to be read from right to left).-Cat's heart. Shows the action of the auricles (Au) and ventricles (V), and also the course of the blood-pressure (Bl. pr.). In the ventricular trace an upward movement of the lever $=$ contraction. In the auricles a downward movement = contraction. The heart was depressed by vagus stimulation at the point ind. cated in the second tracing from the top of the figure. A marked full of blood-pressure ensued. Then (at the points indicated by the mark in the uppermost tracing) single induction shocks were sent into th heart. These shocks cause a more rapid and more effective series of ventricular beats, accompanied by a decided improvement in the blood pressure. The lowest tracing marks time in half-seconds.

Further, even if galvanic and faradic currents were free from the grave dangers to which I have alluded, there would be very little to be said in favour of their use as direct cardiac stimulants in a sudden and urgent crisis. Galvanic and faradic currents, too weak to induce fibrillar contraction in a heart of depressed excita- bility, have a comparatively trivial influence in exciting or accelerating its beat. Wo want a much more effective and speedy mode of exciting rhythmic contraction, and one that will have a direct and powerful influence in calling forth a series of beats in the depressed or inhibited heart, while at the same time free from the danger of throwing the ventricles into delirium. Such a mode of excitation seems to be available in the form of a periodic series of single induction shocks sent through the heart at approximately the normal rate of cardiac action. A single induction shock readily causes a beat in an inhibited heart, and a regular series of induction shocks (for example, sixty or seventy per minute) gives a repular series of heartbeats at the same rate. Never on any occasion have I seen fibrillar contraction excited by such a mode of stimulation. In order to elucidate more fully the influence of a series of induction shocks upon the inhibited heart, I have frequently (in the dog, cat, and rabbit) performed such experiments as the following. The animal being chloroformed, and means being taken to preserve, as far as possible, the normal temperature, the thorax and pericardial sac were laid open; artificial respiration was kept up through a cannula introduced into the trachea. The heart was inhibited by stimulation of the vagus nerve in the neck, and then a periodic series of induction shocks (regulated by a metronome) was applied to the apex of the ventricles. Contraction of the auricles and ventricles was recorded by an adaptation of the graphic method; a blood-pressure tracing was simultaneously made in the usual manner. In this way I was able to obtain an accurate record of the various changes, while at the same time some further information was obtained by direct inspection of the heart. A series of single induction shocks excites a corresponding series of cardiac beats; the ventricular contraction precedes the auricular contraction when the exciting shocks are applied to the ventricles. Fach systole causes the ejection of a considerable amount of blood into the aorta and pulmonary artery, and a marked rise of the blood-pressure at each beat. The mean pressure is raised from the low point to which it had fallen in consequence of the cardiac standstill; it does not, however, attain the normal height, even though a long series of beats is elicited by the stimulating shocks. This fact is due to the feebleness of the auricular contraction under inhibitory influence. For the auricles beat so feebly (in response to the stimulation) that they are unable to pump their contents into the ventricles in the normal vigorous fashion. The ventricles fill very slowly, and, at the moment of contraction, contain much less blood than in the normal state; hence the amount of blood thrown into the aorta in a given space of time is much diminished, and the arterial pressure fails to attain its ordinary height. Nevertheless, the artificially excited beats are decidedly advantageous, inasmuch as they arrest the fall of the blood-pressure and even cause a rise -involving an improvement in the circulatory flow in the coronary system, as well as in the other vessels.

Moreover, the advantages of direct excitation of the heart are not by any means confined to the beneficial effect induced in the blood-pressure; nor to this combined with the obviating of any over-distension of the ventricular cavities with blood. For induction shocks applied to the heart exert an important influence, not only in removing the arrest of rhythmic contraction and restoring the periodic beat, but in counteracting, in a marked degree, the depressing effects of vagus stimulation upon the contraction force of the cardiac muscle. During a period of inhibitory standstill, a cardiac beat excited by a direct stimulus is, as a rule, markedly weakened. But when a regular series of stimuli are employed, the contraction power becomes rapidly improved; the beats increase in force, and often approach the normal strength. Similarly, when the heart has been greatly slowed by inhibitory impulses, the spontaneous beats are frequently reduced in energy; but the application of a periodic series of induction shocks (at approximately the normal rate) leads not only to the manifestation of a regular series of beats at the same rate, but, as a rule, to a pronounced augmentation in the force of the individual beats.

Hence it is evident that, in addition to the improvement in the blood-pressure resulting from direct excitation of the heart by a series of induction shocks, there is also a beneficial effect exercised upon the contractile mechanism of the inhibited heart. The depressing influence exerted through the vagus nerve upon the rhythm and contraction force are in large measure counteracted by direct excitation of the organ. In order that such excitation should be as effective as possible it is probably best to send the stimulating shocks through the whole heart, so that the auricles may come directly under their influence as well as the ventricles. 


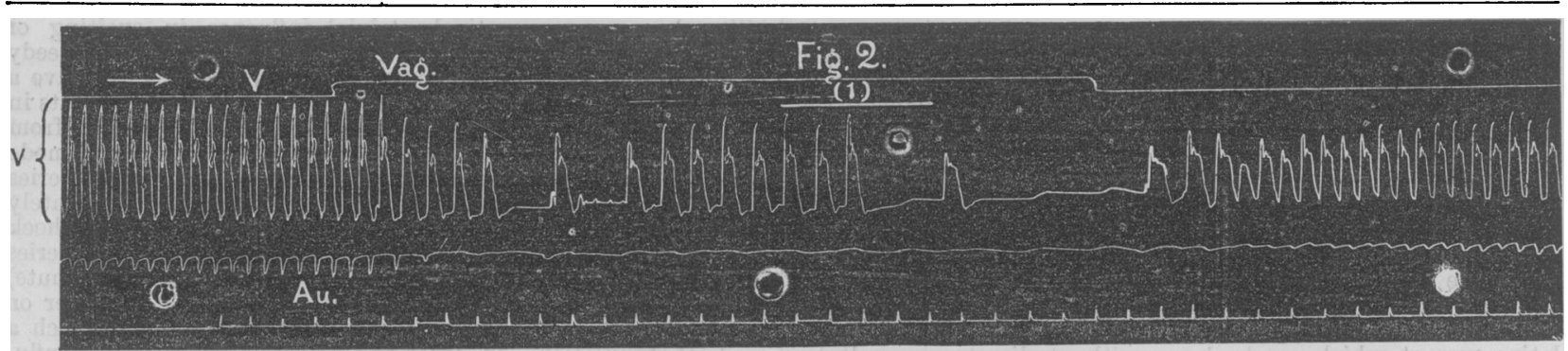

Fig 2 (to be read from leit to right).-Cat's heart. Uppermost tracing shows the period of vagus stimulation. Second tracing (marked V) records the action of the ventricles (upward movement = contraction); and the third tracing (marked Au.) the action of the auricles (downward movement = contraction). The lowest line indicates half-seconds. After the heart had been depressed and enfeebled by vagus stimulation a periontic series of (enght as compared with was applied to the ventricles. The resulting group of beats is marked (1) The indivect of excitation is very apparent.

In order to do this in man one electrode should be applied in front over the area of cardiac impulse, and the other over the region of the fourth dorsal vertebra behind, so that the induction shocks may traverse the organ. The electrodes should be of considerable extent (for example, large sponge electrodes), and they and the skin should be well moistened with salt solution. The shocks employed should be strong, sufficient to excite powerful contraction in the voluntary muscles.

Such a method, it seems to me, is the only rational and effective one for stimulating by direct means the action of a heart which has been suddenly enfeebled or arrested in diastole by causes of a temporary and transient character. Of course, at the same time, the expedient of artificial respiration must by no means be neglected, but, on the contrary, most sedulously attended to.

\section{ON THE ACTION OF CERTAIN DRUGS ON THE PREGNANT UTERUS.}

By LOMBE ATTHILL, M.D.,

President of the King and Queen's College of Physicians, Ex-Master of the Rotunda Hospital, Dublin, etc.

In the number of the British Medical Journal for November 24th a correspondent asks if, in a case of suspected abortion, "he should prescribe a mixture containing iron or any other emmenagogue which causes abortion (the italics are mine), how would he stand if accused of intentionally procuring abortion?" And, again, in the case of a married woman who has " missed one menstrual period and is complaining of 'neuralgia,' how is the practitioner to act in similar obscure cases? Is he justified in giving an emmenagogue?" As I have for more than twenty years carefully watched the action of medicines on the utero-ovarian system, and specially with reference to their effects on the menstrual function, my opportunities for observation having been exceptionally great, and as I take much interest in the question, I venture briefly to make a few remarks on the subject, for I think your correspondent and numberless other practitioners are labouring under a false impression as to the power of drugs in such cases as he refers to.

It is right that I should, in the first instance, say that I have never made any experiments on pregnant women, and that the opinions I now hold are based, first, on the fact that, in the treatment of certain diseases and affections in which I felt myself called on to administer to pregnant women drugs supposed to have the power of inducing abortion, I never observed them to to have any such effect : and, secondly, that having administered the so-called emmenagogues to women who were not pregnant, in almost every possible form, my belief in their value has been so shaken that I have grave doubts as to their efficacy, except in cases where the amenorrhœa depends on the presence of some condition or affection such as anæmia, incipient phthisis, etc., whicn being removed or cured, menstruation recurs.

I will relate a case which made a great impression on me at the time, and has materially influenced my practice ever since. About fifteen years ago I was asked to see a lady threatened with abortion at about the tenth week of pregnancy. She had been married two years, and during that time had aborted on three previous occasions. I found that she had lost some blood, which continued to come away continuously, but there were no pains; and, on examining her, the os uteri proved to be patulous. She was very nervous, having had sharp hæmorrhage on a previous occasion. Taking into consideration her history in connection with her present state, I came to the conclusion that she would again abort, probably with hæmorrhage; and I deemed that the sooner the ovum was expelled the less risk there would be of the occurrence of a dangerous loss. With the view, therefore, of lessening the risk of hæmorrhage and exciting the uterus to expel the ovum, I put her on ergot. The result was most satisfactory, but not what books would have led a student to suppose. No uterine action followed on the administration of the ergot, but the hæmorrhage gradually lessened. I kept this patient in bed for many weeks, and in due time she gave birth to a son, who still lives and is in robust health.

From that time on I have treated similar cases on the same principle-that is, to patients threatened with abortion, hæmorrhage being present, but uterine action, indicated by the occurrence of pains, not having been excited, I administer ergot; these patients are frequently plethoric, with relaxed muscular tissue, and I think it probable that in them the ergot excites a tonic contraction of the muscular tissue of the uterus, which by lessening the blood-supply checks the hæmorrhage. In some the ergot excited uterine action, but in all these I had littls hope of averting abortion, and believed that it was inevitable. In many ergot seemed to produce no effect at all, and my belief is that ergot will rarely excite uterine action in cases of threatened abortion, unless the ovum is already blighted and virtually a foreign body. If, therefore, for any reason, the administration of ergot was deemed necessary in the case of a pregnant woman, I should not hesitate to give it. I may add that for many years I was in the habit of administering ergot for several days before the expected date of parturition to patients liable to post-partum hæmorrhage, with the view of anticipating its occurrence, with very satisfactory results; in no single case did labour set in earlier than was expected, in none did the child suffer in any way, while in two or three cases the occurrence of labour seemed to have been actually delayed by several days. Iron and quinine $I$ have for years administered as freely and in as large doses to pregnant women, as to any other patients, with perfect safety; in fact, I much doubt whether the ordinary medicines given in ordinary doses have any effect on the retro-ovarian system, whether it be in the case of pregnant women, or taken during the continuance of the menstrual flow; but as I have recently, in a paper published in the December number of the Dublin Journal of Medical Science, an abstract of which appeared in the JoURNAL for December 1st, discussed this part of the subject at some length, I abstain from referring further to it now.

I must, however, express my surprise at "Omega's" hesitating to prescribe iron in a case of suppression of the menses, and at his classing it with "emmenagogues which cause abortion." Personally, I know of no emmenagogue which will cause abortion ergot, quinine, and strychnine, in ordinary medicinal doses, I am satisfied, will not do so. Savin and rue, etc., I have never ventured to give to pregnant women, because I believed them to be unsafe drugs to administer in such cases; but iron is perfectly innocuous, and its being classed by any practitioner with "enimenagogues which cause abortion," shows how little consideration has been given to the action of drugs on the utero-ovarian system by medical men.

I can, in conclusion, without hesitation, say that in cases similar to those referred to by "Omega," any of the ordinary tonics, including quinine and strychnine, in ordinary doses, may be safely 